\title{
Transgenerational Transmission of Enhanced Ocular Dominance Plasticity from Enriched Mice to Their Non-enriched Offspring
}

\author{
Evgenia Kalogeraki, ${ }^{1}$ Rashad Yusifov, ${ }^{1,2}$ and ${ }^{1}$ Siegrid Löwel ${ }^{1,3}$
}

https://doi.org/10.1523/ENEURO.0252-18.2018

${ }^{1}$ Department of Systems Neuroscience, J.F.B. Institut für Zoologie und Anthropologie, 37075 Göttingen, ${ }^{2}$ Göttingen Graduate School of Neurosciences, Biophysics and Molecular Biosciences, Universität Göttingen, 37073 Göttingen, Germany, and ${ }^{3}$ Campus Institute for Dynamics of Biological Networks, 37075 Göttingen, Germany

\begin{abstract}
In recent years, evidence has accumulated that non-Mendelian transgenerational inheritance of qualities acquired through experience is possible. In particular, it has been shown that raising rodents in a so-called enriched environment (EE) can not only modify the animals' behavior and increase their susceptibility to activity-dependent neuronal network changes, but also influences both behavior and neuronal plasticity of the non-enriched offspring. Here, we tested whether such a transgenerational transmission can also be observed in the primary visual cortex (V1) using ocular dominance (OD) plasticity after monocular deprivation (MD) as a paradigm. Whereas OD plasticity after $7 \mathrm{~d}$ of MD is absent in standard-cage (SC) raised mice beyond postnatal day (P) 110 , it is present lifelong in EE-raised mice. Using intrinsic signal optical imaging to visualize cortical activity, we confirm these previous observations and additionally show that OD plasticity is not only preserved in adult EE mice but also in their adult non-enriched offspring: mice born to enriched parents, but raised in SCs at least until P110 displayed similar OD shifts toward the open eye after $7 \mathrm{~d}$ of MD as age-matched EE-raised animals. Furthermore, testing the offspring of EE-female versus EE-males with SC-mating partners revealed that only pups of EE-females, but not of EE-males, preserved OD plasticity into adulthood, suggesting that the life experiences of the mother have a greater impact on the continued V1 plasticity of the offspring. The OD plasticity of the non-enriched pups of EE-mothers was, however, mechanistically different from that of non-enriched pups of EE-parents or EE mice.
\end{abstract}

Key words: enriched environment; mouse; ocular dominance plasticity; optical imaging; transgenerational; visual cortex

\section{Significance Statement}

Recently evidence is accumulating that life experiences and thus acquired qualities of parents can be transmitted across generations in a non-Mendelian fashion and have a significant impact on the fitness of offspring. Raising mice in a so-called enriched environment with enhanced opportunities for social interaction, voluntary physical exercise, and explorative behavior has been shown to boost cortical plasticity. Our results now show that the plasticity-promoting effect of enrichment on ocular dominance plasticity, a well-established plasticity paradigm in a primary sensory cortex, can also be transmitted from enriched parents to their non-enriched offspring. Thus cortical plasticity is not only influenced by an animal's life experiences but can also be modified by the life experiences of its parents.

Received June 28, 2018; accepted December 14, 2018; First published January 21, 2019.

The authors declare no competing financial interests.
Author Contributions: E.K. and S.L. designed the research; E.K. and R.Y. performed the experiments and analyzed the data; E.K., S.L., and R.Y. wrote the paper. 


\section{Introduction}

During brain development, experience is continuously interacting with genetic information to shape and functionally optimize neuronal circuits. The environmental conditions under which animals grow up therefore exert a powerful influence on the functioning of their brain and behavior. There is a rich and growing body of evidence documenting changes across molecular, anatomical, and functional levels when animals raised in a so-called enriched environment (EE) are compared with animals housed in standard cages (SCs; Sale et al., 2014). Enriched conditions refer to paradigms in which animals are housed in larger groups in bigger and spatially complex cages equipped with mazes, toys, and running wheels. In this environment, animals experience a "combination of complex inanimate and social stimulation" (Rosenzweig et al., 1962) because of enhanced opportunities to engage in voluntary physical, social, and cognitive stimulation (van Praag et al., 2000; Löwel et al., 2017). In rodents, enrichment alters the expression of key signaling molecules involved in regulating brain excitability and plasticity (Cancedda et al., 2004): BDNF and serotonin levels are increased (Cancedda et al., 2004; Sale et al., 2004, 2007; Baroncelli et al., 2010), GABAergic inhibition is reduced (Greifzu et al., 2014) and the density of extracellular matrix perineuronal nets is reduced (Sale et al., 2007). Furthermore, EE prolongs and restores ocular dominance (OD) plasticity in the visual cortex into old age (Baroncelli et al., 2010; Greifzu et al., 2014, 2016), increases the volume of many brain areas (Diamond et al., 1964), and notably also alters maternal behavior (Sale et al., 2004).

In addition to these "immediate" effects on brain functioning and plasticity, exposure to EE can also influence brain plasticity and behavior of the next generation (Arai and Feig, 2011). Early studies had already documented that exposure of pregnant rats to an EE enhanced not only their ability to find their way in a maze, but also the performance of their offspring in the same task (Kiyono et al., 1985). Furthermore, juvenile enrichment [EE from postnatal day (P)14-P28] not only rescued a genetic defect in long-term potentiation and memory formation in the short-term enriched mice, but also in their 4-week-old non-enriched offspring (Arai et al., 2009). EE during the first days of life was shown to accelerate visual system development because pups of EE-mice receive higher levels of maternal care, continuous physical contact, and more licking compared with SC-reared pups (Cancedda

This work was supported by the Federal Ministry of Education and Research, Germany, Grants 01GQ0921 (E.K.) and 01GQ0810 (S.L.) and by Grants from the Deutsche Forschungsgemeinschaft through the Collaborative Research Center 889 "Cellular Mechanisms of Sensory Processing" to S.L. (Project B5). We thank Matthias Schink for excellent animal care, and Andrew Richard Garrett for helpful comments on this manuscript.

Correspondence should be addressed to Siegrid Löwel at sloewel $@$ gwdg.de.

https://doi.org/10.1523/ENEURO.0252-18.2018

Copyright (C) 2019 Kalogeraki et al.

This is an open-access article distributed under the terms of the Creative Commons Attribution 4.0 International license, which permits unrestricted use, distribution and reproduction in any medium provided that the original work is properly attributed. et al., 2004). Interestingly, the licking-grooming behavior itself is heritable: the offspring of high and low frequent licking-grooming mothers become high and low frequent licking-grooming mothers, respectively (Arai and Feig, 2011).

Motivated by these studies, we analyzed whether there is also transgenerational transmission of increased plasticity in a primary sensory cortex and chose the mouse primary visual cortex (V1) as our model system. OD plasticity in V1 induced by monocular deprivation (MD) is a well established model system to study cortical plasticity (Wiesel and Hubel, 1963). Briefly occluding one eye causes a shift in the OD toward the open eye (Wiesel and Hubel, 1963; Dräger, 1978). In SC-raised mice, OD plasticity is most pronounced in juvenile animals during the critical period (P25-P35), reduced in young adult animals, and not detectable in animals beyond P110 after $7 \mathrm{~d}$ of MD (Lehmann and Löwel, 2008; Espinosa and Stryker, 2012). In contrast, mice raised in EE-cages or transferred to EE after P110 display lifelong OD plasticity (Greifzu et al., 2014, 2016).

Here we tested whether the plasticity-promoting effect of $E E$ on $O D$ plasticity is also present in non-enriched SC-raised pups of EE mice. To this end, pregnant EEmothers were transferred to SCs, the offspring were raised in SCs into adulthood ( $>$ P110), and then OD plasticity was analyzed after $7 \mathrm{~d}$ of $\mathrm{MD}$ using intrinsic signal optical imaging (Cang et al., 2005). During MD, spatial vision of the open eye was quantified daily by optometry (Prusky et al., 2004, 2006). OD plasticity was preserved in the offspring of EE-parents and EE-mothers, but not in offspring of EE-fathers. Whereas the OD shift of nonenriched offspring of EE-parents was mediated by a reduction of $\mathrm{V} 1$ activation through the deprived eye, as typically seen in adult EE-mice (Greifzu et al., 2014; and present study), OD shifts of the offspring of EE-mothers were mechanistically different. Our data clearly show that even in a primary sensory cortex, the plasticity promoting effect of growing up in a stimulating (or less deprived) environment not only affects the mice experiencing this enrichment, but also their non-enriched offspring, and enriched mothers seem to have a bigger impact on the offspring's cortical plasticity.

\section{Materials and Methods}

\section{Animals and rearing conditions}

C57BL/6 mice were obtained from the mouse colony of the central animal facility of our university, and housed with a $12 \mathrm{~h}$ light/dark cycle, with food and water available ad libitum. All experimental procedures comply with $\mathrm{Na}$ tional Institutes of Health guidelines for the use of animals.

Mice were housed in either normal SC or EE cages. EE cages [Marlau, Viewpoint; $56 \times 37 \times 32 \mathrm{~cm}(\mathrm{~L} \times \mathrm{W} \times \mathrm{H})$ ] are approximately nine times larger than our SCs [26 $\times 20$ $\times 14 \mathrm{~cm}[\mathrm{~L} \times \mathrm{W} \times \mathrm{H})]$, with two floors, providing mice a bigger exploration area, more opportunities for social interaction (16 mice compared with 3-4 in SCs) and physical exercise (3 running wheels). The lower compartment is divided in two areas: the "living area" with three running wheels for physical exercise and a red tunnel to protect 
the animals from light during the day, and the "food area" where food is located. To move from the "living area" to the "food area", mice have to go to the upper compartment using the ladder, pass through the maze, and slide down through a tube. They can return to the "living area" through a revolving door which opens only in one direction, thus they are forced to move through the maze again to get food. The maze was changed three times per week, and there were in total 12 different configurations. For the initial comparison of OD plasticity in adult ( $>$ P110) mice, we used either EE- or SC-raised mice. Mice in these groups were born and raised in EE or SC cages until the experiment. For testing whether increased plasticity can be transferred to the next generation, mating of EE-mice took place in EE-cages and pregnant females were then transferred to SCs 5-7 d before delivery.

Furthermore, we crossed EE-males with SC-females as well as SC-males with EE-females. The mating always happened in the cage of the female mouse. Pregnant EE-mothers were transferred to SCs (or stayed in SCs for the SC-mother/EE-father pairing), the offspring were raised in SCs into adulthood ( $>$ P110), and then OD plasticity was analyzed after $7 \mathrm{~d}$ of MD using intrinsic signal optical imaging (Cang et al., 2005).

In summary, the following five experimental groups were analyzed for the present study: (1) SC-raised mice (SC; $n=8$, age range P137-P200), (2) EE-raised mice (EE; $n=8$, age range P161-P168), (3) SC-raised mice from parents raised in EE (EE-parents; $n=10$, age range P118-P261), (4) SC-raised mice from fathers raised in EE and mothers raised in SC (EE-father; $n=10$, age range P127-P194), and (5) SC-raised mice from mothers raised in EE and fathers raised in SC (EE-mother; $n=11$, age range P142-P188; Table 1). Mice within each experimental group were from 2 to 3 litters with different parents and were assigned to $M D$ and noMD conditions to ensure littermate controls.

\section{Monocular deprivation}

The animals' right eye was deprived for $7 \mathrm{~d}$ according to published protocols (Gordon and Stryker, 1996). Briefly, mice were anesthetized using $2 \%$ isoflurane in a mixture of $\mathrm{O}_{2}$ and $\mathrm{N}_{2} \mathrm{O}(1: 1)$, the eyelids were trimmed and sutured together. Mice were returned to their home cages for recovery and checked daily to ensure that the eyes remained closed for the following $7 \mathrm{~d}$.

\section{Virtual reality optomotor test}

To check visual capabilities and the effectiveness of the $M D$, both the spatial frequency and the contrast sensitivity threshold of the optomotor reflex of all mice was measured daily using the virtual reality optomotor system (Prusky et al., 2004). Briefly, freely moving mice were positioned on a small elevated platform surrounded by four computer monitors $(33.5 \times 26.5 \mathrm{~cm})$ forming a box. A rotating virtual cylinder, composed of a vertical sine wave grating, was projected on the screens. Parameters including spatial frequency, contrast and speed of the moving sine wave grating could be varied by the experimenter. On detecting the stimulus, the mouse will reflexively track the grating by moving the head in the direction of rotation.
Table 1 Ages and ODIs of experimental animals

\begin{tabular}{|c|c|c|c|c|}
\hline Figure & Group & Mouse & Age, d & ODI \\
\hline \multirow[t]{16}{*}{1} & SC/noMD & 1 & 137 & 0.34 \\
\hline & & 2 & 139 & 0.33 \\
\hline & & 3 & 151 & 0.27 \\
\hline & & 4 & 200 & 0.31 \\
\hline & SC/MD & 1 & 152 & 0.19 \\
\hline & & 2 & 154 & 0.30 \\
\hline & & 3 & 155 & 0.23 \\
\hline & & 4 & 195 & 0.37 \\
\hline & EE/noMD & 1 & 164 & 0.32 \\
\hline & & 2 & 161 & 0.26 \\
\hline & & 3 & 161 & 0.20 \\
\hline & & 4 & 168 & 0.20 \\
\hline & EE/MD & 1 & 163 & -0.01 \\
\hline & & 2 & 164 & 0.12 \\
\hline & & 3 & 164 & 0.07 \\
\hline & & 4 & 164 & -0.03 \\
\hline \multirow[t]{10}{*}{2} & EE-parents/noMD & 1 & 119 & 0.32 \\
\hline & & 2 & 125 & 0.24 \\
\hline & & 3 & 209 & 0.17 \\
\hline & & 4 & 205 & 0.23 \\
\hline & EE-parents/MD & 1 & 118 & 0.11 \\
\hline & & 2 & 126 & 0.10 \\
\hline & & 3 & 130 & 0.16 \\
\hline & & 4 & 261 & 0.07 \\
\hline & & 5 & 200 & 0.05 \\
\hline & & 6 & 208 & 0.13 \\
\hline \multirow[t]{21}{*}{3} & EE-fathers/noMD & 1 & 127 & 0.16 \\
\hline & & 2 & 135 & 0.24 \\
\hline & & 3 & 136 & 0.21 \\
\hline & & 4 & 188 & 0.25 \\
\hline & & 5 & 184 & 0.32 \\
\hline & EE-fathers/MD & 1 & 132 & 0.19 \\
\hline & & 2 & 132 & 0.12 \\
\hline & & 3 & 133 & 0.20 \\
\hline & & 4 & 136 & 0.20 \\
\hline & & 5 & 194 & 0.23 \\
\hline & EE-mothers/noMD & 1 & 142 & 0.26 \\
\hline & & 2 & 143 & 0.33 \\
\hline & & 3 & 168 & 0.28 \\
\hline & & 4 & 169 & 0.22 \\
\hline & & 5 & 151 & 0.21 \\
\hline & & 6 & 188 & 0.27 \\
\hline & EE-mothers/MD & 1 & 166 & 0.16 \\
\hline & & 2 & 174 & 0.15 \\
\hline & & 3 & 175 & 0.11 \\
\hline & & 4 & 176 & 0.04 \\
\hline & & 5 & 177 & 0.15 \\
\hline
\end{tabular}

Age and ODI of all experimental animals of each experimental group, subdivided by figure in which data were displayed.

Spatial frequency thresholds at full contrast and contrast thresholds at six different spatial frequencies [0.031, $0.064,0.092,0.103,0.192,0.272$ cycles/degree (cyc/ deg)] were measured daily, before and after MD (7 d total).

\section{Optical imaging of intrinsic signals and visual stimuli Surgery}

Mice were anesthetized with $2 \%$ halothane in $\mathrm{O}_{2}: \mathrm{N}_{2} \mathrm{O}$ $(1: 1)$ and injected with rimadyl $(0.1 \mathrm{mg} /$ mouse, s.c.; Pfizer), atropine (0.3 mg/mouse, s.c.; Franz Köhler), dexamethasone ( $0.2 \mathrm{mg} / \mathrm{mouse}$, s.c.; Ratiopharm), and chlorprothixene (0.2 mg/mouse, i.m.; Sigma-Aldrich). After 
placing animals in a stereotaxic frame, anesthesia was maintained with $0.8 \%$ halothane in a mixture of $\mathrm{O}_{2}: \mathrm{N}_{2} \mathrm{O}$ (1:1). A small incision of the skin was made to expose visual cortex and low-melting point agarose $(2.5 \%$ in $0.9 \% \mathrm{NaCl}$ ) and a glass coverslip were placed over the exposed area. In case of an MD mouse, the sutures were removed to open the deprived eye.

\section{Data acquisition and visual stimulation}

Mouse V1 responses were recorded through the skull using the "Fourier"-imaging method (Kalatsky and Stryker, 2003), optimized for the assessment of OD plasticity (Cang et al., 2005). V1 signals were visualized with a CCD-camera (Dalsa 1M30) using a $135 \times 50 \mathrm{~mm}$ tandem lens configuration (Nikon), with red illumination light (610 $\pm 10 \mathrm{~nm}$ ). Active brain regions absorb more of the red light and appear darker in the images. Frames were acquired at a rate of $30 \mathrm{~Hz}$, temporally binned to $7.5 \mathrm{~Hz}$, and stored as $512 \times 512$ pixel images after spatial binning of the camera image.

Visual stimuli were presented on a high refresh rate monitor (Hitachi, ACCUVUE, HM-4921-D, 21 inch) positioned $25 \mathrm{~cm}$ from the eyes. Stimuli consisted of white drifting horizontal bars ( $2^{\circ}$ wide). The amplitude component of the optical signal represents the intensity of neuronal activation (expressed as fractional change in reflectance $-10^{-4}$ ) and was used to calculate OD. At least three maps per animal were averaged to compute the OD index $(\mathrm{ODI})$ as $(\mathrm{C}-\mathrm{I}) /(\mathrm{C}+\mathrm{I})$, with $\mathrm{C}$ and I representing the response magnitudes of each pixel to visual stimulation of the contralateral and ipsilateral eye. The ODI ranges from -1 to +1 , with negative values representing ipsilateral and positive values representing contralateral dominance. Note that the V1 activity maps illustrated in the results section are the averages of at least three maps from individual animals. OD maps were always assessed and quantified by an experimenter blind to the animals' or its parents' rearing conditions.

\section{Statistical analysis}

All intragroup and intergroup comparisons were analyzed by ANOVA followed by Bonferroni post hoc test. The intergroup comparison of the enhancement of the spatial frequency and contrast sensitivity thresholds were analyzed by two-way ANOVA with repeated measurements and Bonferroni correction. Normal distribution of data was checked using Shapiro-Wilk test. Data were analyzed using GraphPad Prism 7 and IBM SPSS statistics. The levels of significance were set as $* p<0.05$, $* * p<0.01$, $* * * p<0.001$. Data are represented as means \pm SEM (Table 2).

\section{Results}

\section{OD plasticity is preserved in adult EE-raised but not in adult SC-raised mice}

It was previously reported that OD plasticity is an agedependent process (Espinosa and Stryker, 2012) because SC-raised mice no longer exhibit OD shifts after $7 \mathrm{~d}$ of MD when they are older than P110 (Lehmann and Löwel, 2008). In contrast, mice raised in EE display lifelong OD plasticity (Greifzu et al., 2014, 2016). Here, we confirm these previous observations by comparing OD plasticity after $7 \mathrm{~d}$ of $\mathrm{MD}$ in adult mice raised in either SCs (age range P137-P200) or EE cages (age range P161-P168; Fig. 1) using intrinsic signal optical imaging (Cang et al., 2005). In SC-mice, V1 was dominated by the contralateral eye, and remained dominated by the contralateral (deprived) eye also after MD (contralateral V1 activation noMD/MD: $1.45 \pm 0.08 / 1.65 \pm 0.13, p=0.907$, ANOVA; ipsilateral V1 activation noMD/MD: $0.77 \pm 0.04 / 1.06 \pm$ $0.15, p=0.6298$, ANOVA). In contrast, in EE-mice, V1 activation through the (previously) deprived eye was significantly reduced after MD (contralateral noMD/MD: 2.22 $\pm 0.14 / 1.74 \pm 0.11, p=0.034$, ANOVA; ipsilateral noMD/ MD: $1.49 \pm 0.08 / 1.68 \pm 1.22 ; p=0.917$, ANOVA; Fig. $1 A, C)$. Accordingly, the average ODI of SC-mice without MD was $0.31 \pm 0.02(n=4)$, and did not change after MD (ODI: $0.27 \pm 0.04, n=4 ; p=0.38$, ANOVA). In contrast, age-matched EE-mice showed a significant OD shift after MD: the average ODI of adult EE-mice decreased from $0.25 \pm 0.03(n=4)$ without MD to $0.04 \pm 0.03$ after MD $(n=4 ; p=0.0037$, ANOVA; Fig. 1B), and average ODI values after $M D$ were significantly different between SCand EE-mice ( $p=0.001$, ANOVA). Notably, the OD shift of EE-mice was mediated by a reduction of $\mathrm{V} 1$ activation after visual stimulation of the contralateral eye, as shown in previous publications on EE-mice (Greifzu et al., 2014).

\section{OD plasticity was present in non-enriched offspring of EE-parents}

Next, we visualized OD plasticity in V1 of the nonenriched offspring of EE-parents, i.e., in mice that were born and raised in SCs until at least P119, and thus never experienced EE-conditions on their own (see Materials and Methods for a detailed explanation of mating conditions). To trigger plasticity, half of the mice were subjected to $7 \mathrm{~d}$ of $\mathrm{MD}$, whereas control mice from the same litter did not receive MD. As expected, V1 of mice without MD was dominated by the contralateral eye. In contrast, V1 of MD-mice was more equally activated by both eyes, and thus showed an OD shift (Fig. 2): in no-MD mice, the two-dimensional OD map showed warm colors, indicating contralateral dominance, whereas in MD-mice, colder colors prevailed the OD map and the OD histogram was shifted to the left (Fig. 2A).

Quantitative analysis of the ODI revealed that the adult non-EE offspring of EE-parents displayed an OD shift in V1 after MD implying that the plasticity promoting effect of EE can be transferred to the next generation (Fig. 2B). Specifically, non-EE mice from EE-parents had a mean ODI of $0.26 \pm 0.02$ ( $n=4, \mathrm{P} 119-\mathrm{P} 209)$, reduced to 0.10 \pm 0.02 after $7 \mathrm{~d}$ of $\mathrm{MD}(n=6, \mathrm{P} 118-\mathrm{P} 261 ; p=0.0019$, ANOVA). As expected, the binocular part of $\mathrm{V} 1$ in mice without MD was activated more strongly after contralateral than after ipsilateral eye stimulation $(p=0.0062$, ANOVA), whereas after MD, the V1 activation was similar after each eye stimulation ( $p>0.999$, ANOVA). The average ODI of monocularly deprived mice of the EE-parents group was significantly lower than that of the MD mice of the SC group (Fig. 1; $0.27 \pm 0.04, n=4, p=0.0019, t$ test). Notably, the observed OD shift was mediated by a 
Table 2 Statistical analysis

\begin{tabular}{|c|c|c|c|c|c|c|c|c|}
\hline Fig. & Group & Parameter & $N$ & $\mathrm{Cl}_{95}$ & Data structure & Comparison & Type of test & $p$ value \\
\hline \multirow[t]{4}{*}{$1 B$} & SC/EE & [A] SC-no MD & 4 & $(0.2632 ; 0.3618)$ & Normal distribution & $A$ vs $B$ & Two-way ANOVA with Bonferroni's & 0.3837 \\
\hline & & [B] SC-MD & 4 & $(0.1463 ; 0.3987)$ & Normal distribution & C vs D & multiple comparisons & $0.0037 * *$ \\
\hline & & [C] EE-no MD & 4 & $(0.1536 ; 0.3364)$ & Normal distribution & $B$ vs $D$ & & $0.001 * *$ \\
\hline & & [D] EE-MD & 4 & $(-0.0738 ; 0.1488)$ & Normal distribution & & & \\
\hline \multirow[t]{8}{*}{$1 C$} & SC/EE & [A] SC-no MD-Contra(C) & 4 & $(1.179 ; 1.716)$ & Normal distribution & $A$ vs $B$ & Mixed ANOVA with Bonferroni's & $0.0064 * *$ \\
\hline & & [B] SC-no MD-Ipsi (I) & 4 & $(0.6298 ; 0.9052)$ & Normal distribution & $A$ vs $C$ & multiple comparisons & 0.9071 \\
\hline & & [C] SC-MD-Contra (C) & 4 & $(1.248 ; 2.052)$ & Normal distribution & $B$ vs $D$ & & 0.6289 \\
\hline & & [D] SC-MD-Ipsi (I) & 4 & $(0.5893 ; 1.526)$ & Normal distribution & $C$ vs $D$ & & $0.0228 *$ \\
\hline & & [E] EE-no MD-Contra(C) & 4 & $(1.778 ; 2.666)$ & Normal distribution & E vs $F$ & & $0.003 * *$ \\
\hline & & [F ] EE-no MD-Ipsi (I) & 4 & $(1.25 ; 1,73)$ & Normal distribution & E vs $G$ & & $0.0343 *$ \\
\hline & & [G] EE-MD-Contra (C) & 4 & $(1.41 ; 2.08)$ & Normal distribution & $\mathrm{F}$ vs $\mathrm{H}$ & & 0.9179 \\
\hline & & [H] EE-MD-Ipsi (I) & 4 & $(1.219 ; 2.156)$ & Normal distribution & G vs $\mathrm{H}$ & & $>0.999$ \\
\hline \multirow[t]{2}{*}{$2 B$} & no $\mathrm{MD} / \mathrm{MD}$ & {$[\mathrm{A}]$ no $\mathrm{MD}$} & 4 & $(0.1491 ; 0.3459)$ & Normal distribution & $A$ vs $B$ & Unpaired $t$ test & $0.0019 * *$ \\
\hline & & {$[\mathrm{B}] \mathrm{MD}$} & 6 & $(0.06253 ; 0.1451)$ & Normal distribution & & & \\
\hline \multirow[t]{4}{*}{$2 C$} & no $\mathrm{MD} / \mathrm{MD}$ & [A] no MD-Contra (C) & 4 & $(1.604 ; 2.424)$ & Normal distribution & $A$ vs $B$ & Two-way ANOVA with Bonferroni's & $0.0062 * *$ \\
\hline & & [B] no MD-Ipsi (I) & 4 & $(0.8888 ; 1.506)$ & Normal distribution & $A$ vs $C$ & multiple comparisons & $0.0237 *$ \\
\hline & & [C] MD-Contra (C) & 6 & $(1.055 ; 1.718)$ & Normal distribution & B vs $D$ & & 0.997 \\
\hline & & [D] MD-Ipsi(I) & 6 & $(0.899 ; 1.581)$ & Normal distribution & $C$ vs $D$ & & $>0.999$ \\
\hline \multirow[t]{4}{*}{$3 B$} & EE fathers/ & [A] EE fathers-no MD & 5 & $(0.1633 ; 0.3087)$ & Normal distribution & $A$ vs $B$ & Two-way ANOVA with Bonferroni's & 0.8101 \\
\hline & EE mothers & [B] EE fathers-MD & 5 & $(0.1373 ; 0.2387)$ & Normal distribution & C vs $D$ & multiple comparisons & $0.0011 * *$ \\
\hline & & [C] EE mothers-no MD & 6 & $(0.216 ; 0.3074)$ & Normal distribution & & & \\
\hline & & [D] EE mothers- MD & 5 & $(0.06029 ; 0.1837)$ & Normal distribution & & & \\
\hline \multirow[t]{8}{*}{$3 C$} & EE fathers/ & [A] EE fathers-no MD-Contra(C) & 5 & $(1.25 ; 2.478)$ & Normal distribution & $A$ vs $B$ & Mixed ANOVA with Bonferroni's & $0.0026 * *$ \\
\hline & EE mothers & [B] EE fathers-no MD-Ipsi (I) & 5 & $(0.7768 ; 1.655)$ & Normal distribution & $A$ vs $C$ & multiple comparisons & 0.9850 \\
\hline & & [C] EE fathers-MD-Contra (C) & 5 & $(0.712 ; 2.504)$ & Normal distribution & B vs $D$ & & 0.9689 \\
\hline & & [D] EE fathers-MD-Ipsi (I) & 5 & $(0.6932 ; 1.759)$ & Normal distribution & $C$ vs $D$ & & 0.0537 \\
\hline & & [E]EE mothers-no MD-Contra(C) & 6 & $(1.519 ; 2.101)$ & Normal distribution & E vs $F$ & & $<0.0001 * * *$ \\
\hline & & [F ]EE mothers-no MD-Ipsi (I) & 6 & $(0.9971 ; 1.536)$ & Normal distribution & E vs $G$ & & 0.9854 \\
\hline & & [G] EE mothers-MD-Contra (C) & 5 & $(1.351 ; 2.757)$ & Normal distribution & $\mathrm{F}$ vs $\mathrm{H}$ & & 0.0515 \\
\hline & & [H] EE mothers-MD-Ipsi (I) & 5 & $(1.202 ; 2.266)$ & Normal distribution & G vs $H$ & & 0.9495 \\
\hline \multirow[t]{2}{*}{$4 A$} & EE parents & {$[\mathrm{A}]$ no $\mathrm{MD}$} & 4 & $(0.379 ; 0.3823)$ & Normal distribution & $A$ vs $B$ & One-way ANOVA & $0.0011 * *$ \\
\hline & & {$[B] \mathrm{MD}$} & 6 & $(0.3985 ; 0.437)$ & Normal distribution & & & \\
\hline \multirow[t]{2}{*}{$4 A$} & EE fathers & {$[\mathrm{A}]$ no $\mathrm{MD}$} & 5 & $(0.3792 ; 0.3801)$ & Normal distribution & $A$ vs $B$ & One-way ANOVA & $0.0007 * * *$ \\
\hline & & {$[\mathrm{B}] \mathrm{MD}$} & 5 & $(0.4025 ; 0.4498)$ & Normal distribution & & & \\
\hline \multirow[t]{2}{*}{$4 A$} & EE mothers & {$[\mathrm{A}]$ no $\mathrm{MD}$} & 5 & $(0.387 ; 0.391)$ & Normal distribution & $A$ vs $B$ & One-way ANOVA & $0.0043 * *$ \\
\hline & & {$[B] \mathrm{MD}$} & 5 & $(0.3985 ; 0.4297)$ & Assume normality & & & \\
\hline $4 B$ & EE parents/ EE fathers/ & {$[\mathrm{A}]$ EE parents MD } & 6 & $(4.518 ; 14.58)$ & Normal distribution & $A$ vs $B$ & One-way ANOVA & $>0.999$ \\
\hline & EE mothers & [B] EE fathers MD & 5 & $(5.862 ; 18.28)$ & Normal distribution & $A$ vs $C$ & & $>0.999$ \\
\hline & & [C] EE mothers MD & 5 & $(3.593 ; 11.68)$ & Normal distribution & B vs $\mathrm{C}$ & & 0.4994 \\
\hline $4 C$ & EE parents- & [A] Day 0 & 4 & $(4.368 ; 12.56)$ & Normal distribution & $A$ vs $B$ at 0.031 & Two-way ANOVA with Bonferroni's & $>0.999$ \\
\hline & no MD & [B] Day 7 & 4 & $(4.406 ; 12.68)$ & Normal distribution & $A$ vs $B$ at 0.064 & multiple comparisons & $>0.999$ \\
\hline & & & & & & $A$ vs $B$ at 0.092 & & $>0.999$ \\
\hline & & & & & & $A$ vs $B$ at 0.103 & & $>0.999$ \\
\hline & & & & & & $A$ vs $B$ at 0.192 & & $>0.999$ \\
\hline & & & & & & $A$ vs $B$ at 0.272 & & $>0.999$ \\
\hline $4 D$ & EE parents-MD & [A] Day 0 & 6 & $(4.266 ; 12.42)$ & Normal distribution & $A$ vs $B$ at 0.031 & Two-way ANOVA with Bonferroni's & 0.7252 \\
\hline & & [B] Day 7 & 6 & $(5.582 ; 24.46)$ & Normal distribution & $A$ vs $B$ at 0.064 & multiple comparisons & $<0.0001 * * *$ \\
\hline & & & & & & $A$ vs $B$ at 0.092 & & $<0.0001 * * *$ \\
\hline & & & & & & $A$ vs $B$ at 0.103 & & $<0.0001 * * *$ \\
\hline & & & & & & $A$ vs $B$ at 0.192 & & $<0.0001 * * *$ \\
\hline & & & & & & $A$ vs $B$ at 0.272 & & $>0.999$ \\
\hline $4 E$ & EE fathers- & [A] Day 0 & 5 & $(4.211 ; 12.99)$ & Normal distribution & $A$ vs $B$ at 0.031 & Two-way ANOVA with Bonferroni's & $>0.999$ \\
\hline & no MD & [B] Day 7 & 5 & $(4.229 ; 13.12)$ & Normal distribution & $A$ vs $B$ at 0.064 & multiple comparisons & $>0.999$ \\
\hline & & & & & & $A$ vs $B$ at 0.092 & & $>0.999$ \\
\hline & & & & & & $A$ vs $B$ at 0.103 & & $>0.999$ \\
\hline & & & & & & $A$ vs $B$ at 0.192 & & $>0.999$ \\
\hline & & & & & & $A$ vs $B$ at 0.272 & & $>0.999$ \\
\hline $4 F$ & EE fathers- & [A] Day 0 & 5 & $(4.14 ; 12.83)$ & Normal distribution & $A$ vs $B$ at 0.031 & Two-way ANOVA with Bonferroni's & 0.1166 \\
\hline & MD & [B] Day 7 & 5 & $(5.347 ; 23.99)$ & Normal distribution & $A$ vs $B$ at 0.064 & multiple comparisons & $<0.0001 * * *$ \\
\hline & & & & & & $A$ vs $B$ at 0.092 & & $<0.0001 * * *$ \\
\hline & & & & & & $A$ vs $B$ at 0.103 & & $<0.0001 * * *$ \\
\hline & & & & & & $A$ vs $B$ at 0.192 & & $<0.0001 * * *$ \\
\hline & & & & & & $A$ vs $B$ at 0.272 & & 0.2105 \\
\hline $4 G$ & EE mothers- no MD & [A] Day 0 & 6 & $(4.061 ; 14.5)$ & Normal distribution & $A$ vs $B$ at 0.031 & Two-way ANOVA with Bonferroni's & $>0.999$ \\
\hline & & [B] Day 7 & 6 & $(4.024 ; 14,53)$ & Normal distribution & $A$ vs $B$ at 0.064 & multiple comparisons & $>0.999$ \\
\hline & & & & & & $A$ vs $B$ at 0.092 & & $>0.999$ \\
\hline & & & & & & $A$ vs $B$ at 0.103 & & $>0.999$ \\
\hline & & & & & & $A$ vs $B$ at 0.192 & & $>0.999$ \\
\hline & & & & & & $A$ vs $B$ at 0.272 & & $>0.999$ \\
\hline & & & & & & & & \\
\hline
\end{tabular}


Table 2 Continued

\begin{tabular}{|c|c|c|c|c|c|c|c|c|}
\hline Fig. & Group & Parameter & $N$ & $\mathrm{Cl}_{95}$ & Data structure & Comparison & Type of test & $p$ value \\
\hline \multirow[t]{6}{*}{$4 H$} & EE mothers- & {$[\mathrm{A}]$ Day 0} & 5 & $(4.076 ; 14.61)$ & Normal distribution & $A$ vs $B$ at 0.031 & Two-way ANOVA with Bonferroni's & 0.2940 \\
\hline & MD & [B] Day 7 & 5 & $(5.064 ; 21.3)$ & Normal distribution & $A$ vs $B$ at 0.064 & multiple comparisons & $<0.0001 * * *$ \\
\hline & & & & & & $A$ vs $B$ at 0.092 & & $<0.0001 * * *$ \\
\hline & & & & & & $A$ vs $B$ at 0.103 & & $<0.0001 * * *$ \\
\hline & & & & & & $A$ vs $B$ at 0.192 & & $<0.0001 * * *$ \\
\hline & & & & & & $A$ vs $B$ at 0.272 & & $>0.3072$ \\
\hline
\end{tabular}

The columns in the table from left to right refer to the figures, the groups compared, parameters analyzed, number of animals $(N)$, lower and upper $95 \%$ confidence interval of the mean $\left(\mathrm{Cl}_{95}\right)$, distribution of the values (data structure), comparisons of (sub)groups abbreviated as indicated in the parameter column (comparison), test applied for the comparison, and statistical readout ( $p$ value). Significance levels were set as $* p<0.05, * * p<0.01, * * * p<0.001$.

reduction of deprived (contralateral) eye responses in $\mathrm{V} 1$ : V1 activation after contralateral eye stimulation was 2.02 \pm 0.13 in mice without MD, and decreased to $1.39 \pm 0.13$ after MD ( $p=0.0237$, ANOVA). In contrast, open (ipsilateral) eye responses remained unchanged between no-MD and MD mice (no-MD/MD: $1.20 \pm 0.10 / 1.24 \pm 0.13, p=$ 0.997, ANOVA; Fig. 2C).

\section{OD plasticity was preserved in the offspring of EE- mothers, but not of EE-fathers}

Next, we were interested in investigating whether both parents contribute equally to the observed transgenerational plasticity. To do this, we arranged matings between EE-males and SC-females, and between SC-males and EE-females. Offspring of both pairings were born and
A
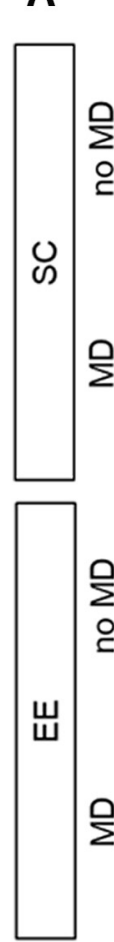
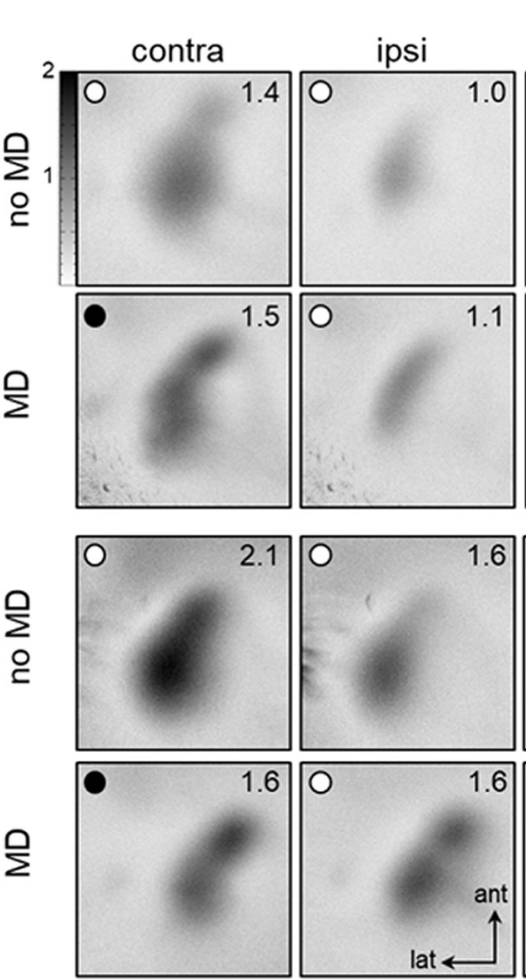

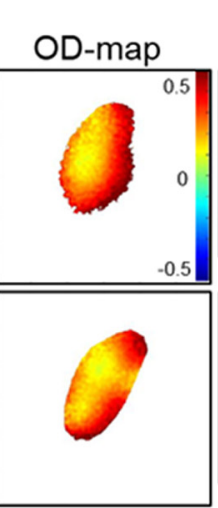

histogram of $(\mathrm{C}-1) /(\mathrm{C}+1)$

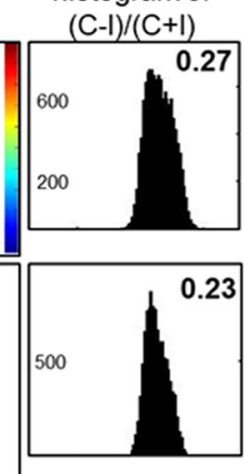

\section{B}
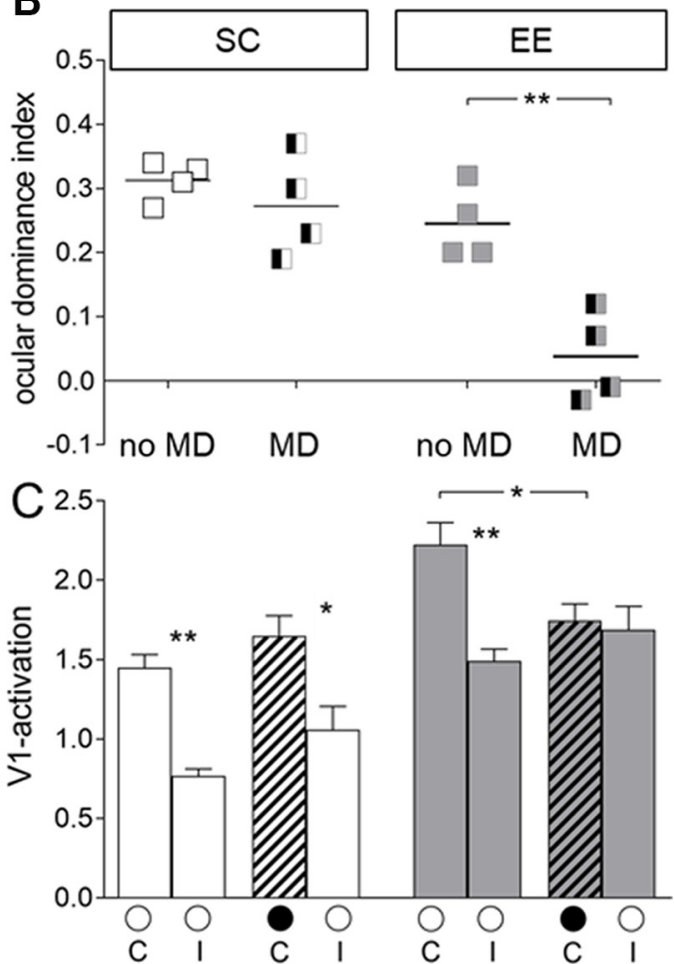

Figure 1. OD plasticity is preserved in V1 of old EE-mice. A, Examples of optically recorded activity maps induced by visual stimulation of the contralateral and ipsilateral eye in the binocular part of V1 of SC- and EE-raised mice, without (no MD) or after 7 $\mathrm{d}$ of MD. Gray scale-coded activity maps [numbers in the top right corner correspond to the quantified $\mathrm{V} 1$ activation $\left(\times 10^{-4}\right)$; see gray scale, left], color-coded two-dimensional OD maps (color codes ODI; see scale to the right of OD map), and the histogram of OD scores, including the average ODI, are illustrated. MD eye is indicated by the black circle in the V1 map, open circles indicate an open eye. In both SC- and EE-mice without MD (no MD), the activity patch evoked by visual stimulation of the contralateral eye is darker than the one of the ipsilateral eye, warm colors prevail in the two-dimensional OD maps and ODI values are positive. After MD, V1 activation changes in EE mice but not in SC mice. Whereas V1 of SC mice remained dominated by the deprived (contralateral) eye, there was an OD shift toward the open eye in V1 of EE mice: after MD, V1 of EE mice was now less strongly activated by the contralateral eye so that both eyes activated V1 similarly, colder colors appeared in the OD maps, and the ODI values were lower, i.e., the ODI histograms shifted to the left (blue arrow). ant, Anterior; lat, lateral. Scale bar, $1 \mathrm{~mm}$. $\boldsymbol{B}$, $\boldsymbol{C}$, Quantification of visual cortical activation before and after MD. ODI $(\boldsymbol{B})$ and V1 activation $(\boldsymbol{C})$ are illustrated. $\boldsymbol{B}$, Optically imaged ODIs without (no MD) and with MD: symbols represent ODI values of individuals, means are marked by horizontal lines. MD is indicated by half-black squares. $\boldsymbol{C}$, V1 activation elicited by stimulation of the contralateral (C) or ipsilateral (I) eye. Hatched bar indicates MD eye. Data represented as mean \pm SEM. Statistical significance was calculated using ANOVA and $p$ values were corrected for multiple comparisons. $* p<0.05, * * p$ $<0.01$. 
A

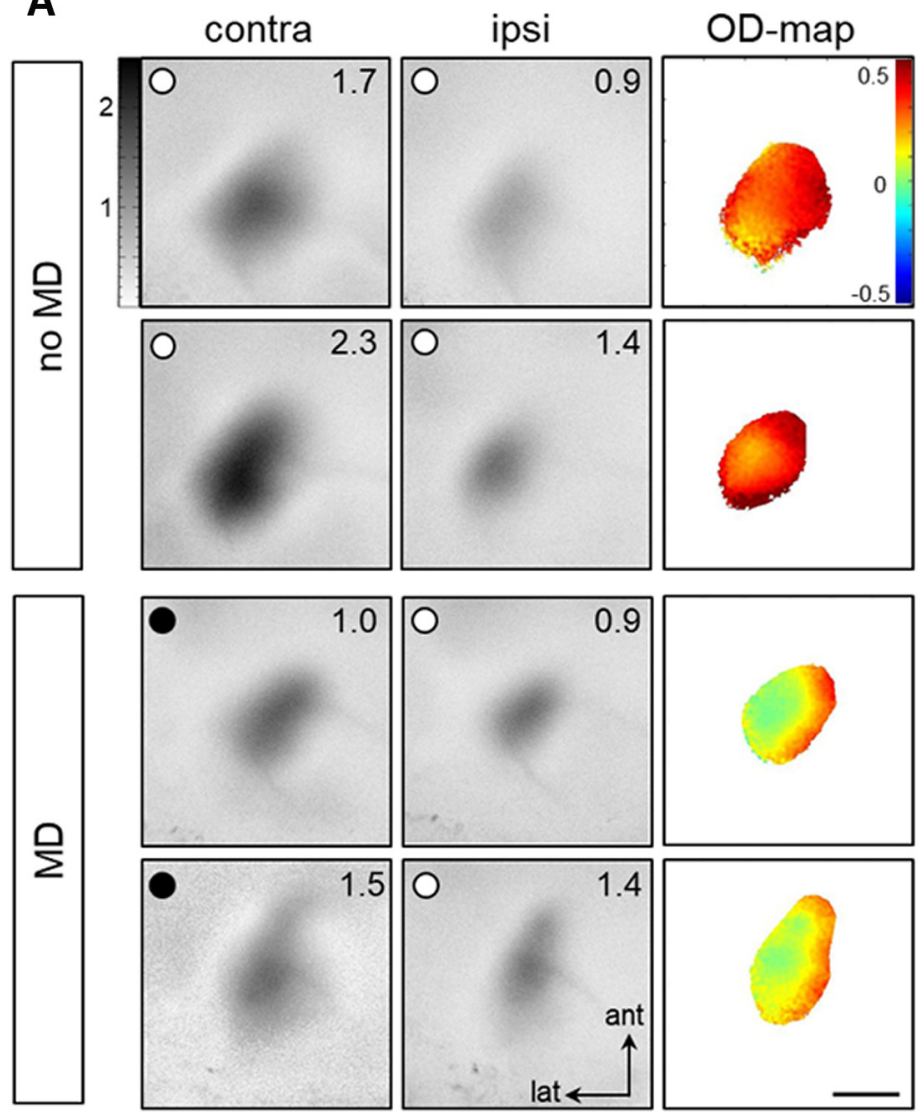

histogram of

$(\mathrm{C}-\mathrm{I}) /(\mathrm{C}+\mathrm{I})$
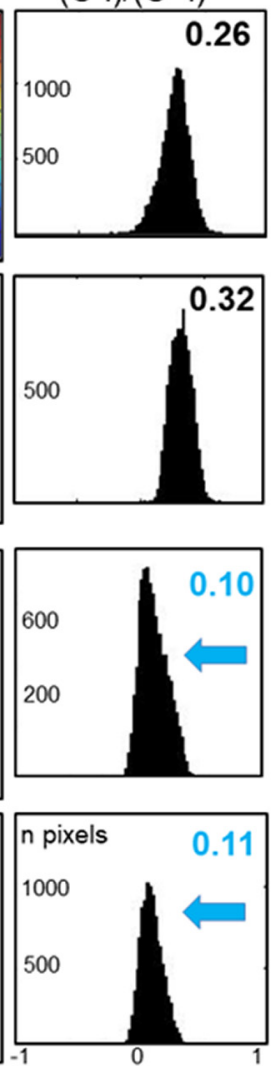

B

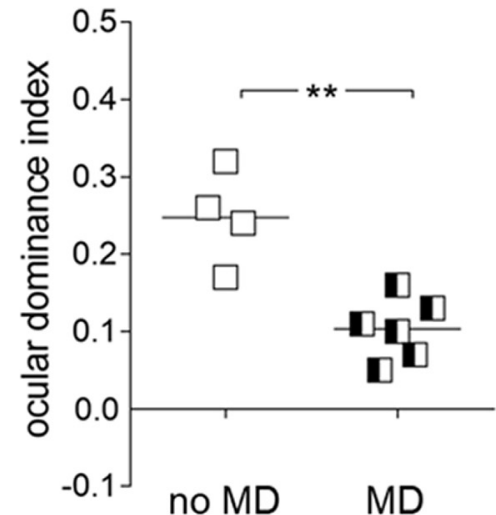

C

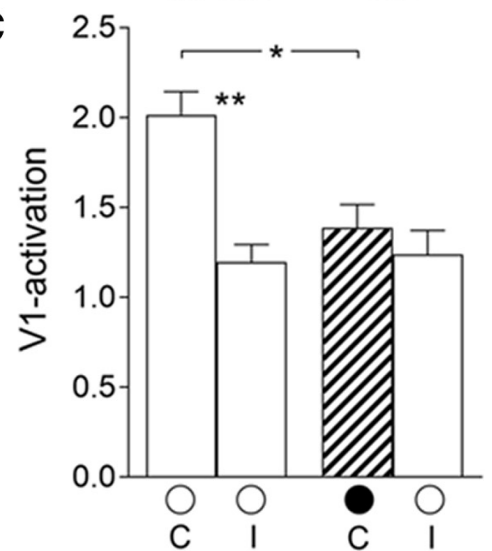

Figure 2 . Transgenerational transmission of enhanced OD plasticity from EE mice to their non-enriched SC-raised offspring. $\boldsymbol{A}$, Optically recorded activity maps of the contralateral (contra) and ipsilateral (ipsi) eye in the binocular part of V1 of SC-raised offspring of EE-parents without and with $7 \mathrm{~d}$ of MD. Data display and quantification as in Figure 1. V1 activity maps from two individual animals of each condition (no MD/MD) are illustrated. In mice without MD (no MD, top 2 rows), the activity patch evoked by visual stimulation of the contralateral eye is darker than the one of the ipsilateral eye, warm colors prevail in the two-dimensional OD maps and ODI values are positive. MD (bottom 2 rows) resulted in an OD shift toward the open eye so that both eyes activated V1 more similarly strong, colder colors appeared in the OD maps, and the ODI values were lower, i.e., the ODI histograms shifted to the left (blue arrows). B, C, Quantification of visual cortical activation before and after MD. ODI (B) and V1 activation $(\boldsymbol{C})$ are illustrated. $* p<0.05$, $* * p<0.01$.

raised in SCs until at least $\mathrm{P} 127$. Thereafter, OD plasticity was assessed by intrinsic signal optical imaging as before.

As expected, visual stimulation showed that the binocular part of V1 of non-deprived adult offspring of both pairings (EE-mother/SC-father and SC-mother/EE-father) was dominated by the contralateral eye (Fig. 3): V1 activation was always stronger after contralateral eye stimulation compared with ipsilateral eye stimulation, the average ODI was positive, and warm colors prevailed in the two-dimensional OD maps. After MD of the contralateral eye in non-enriched mice born to EE-father/SCmother pairings, the ODI did not change: the ODI remained positive, and warm colors continued to dominate the two-dimensional OD map. In contrast, in nonenriched mice born to EE-mother/SC-father pairings, a 7 d MD induced OD shifts: V1 was activated more equally strongly by visual stimulation of either eye, the ODI values were closer to zero, colder colors appeared in the OD map, and the OD histogram shifted to the left (Fig. $3 A$ ).
Specifically, non-enriched offspring of EE-fathers displayed an ODI of $0.24 \pm 0.03(n=5$, P127-P188) which was not significantly changed after MD $(0.19 \pm 0.02 ; n=$ 5, P132-P194; $p=0.81$ ANOVA). In contrast, nonenriched offspring of EE-mothers displayed OD plasticity: the ODI was $0.26 \pm 0.02(n=6, \mathrm{P} 142-\mathrm{P} 188)$, and reduced to $0.12 \pm 0.02$ after MD $(n=5$, P166-P177; $p=$ 0.0011 , ANOVA; Fig. 3B). Furthermore, V1 responses after stimulation of each eye were quantified (Fig. 2C). In nonenriched offspring of EE-father/SC-mother pairings, V1 activation after both contralateral (contra) and ipsilateral (ipsi) eye stimulation did not change after MD (contra, without/with MD: $1.83 \pm 0.18 / 1.61 \pm 0.32 ; p=0.985$, ANOVA; ipsi, without/with MD: $1.24 \pm 0.13 / 1.23 \pm 0.19$, $\mathrm{p}=0.969$, ANOVA). In non-enriched offspring of EEmother/SC-father pairs, V1 activation after contralateral eye stimulation was $1.81 \pm 0.11$ without MD and $2.05 \pm$ 0.25 with MD ( $p=0.985$, ANOVA), whereas V1 activation after ipsilateral eye stimulation was increased from $1.27 \pm$ 0.10 without MD to $1.73 \pm 0.19$ after MD. Although this 
A

A
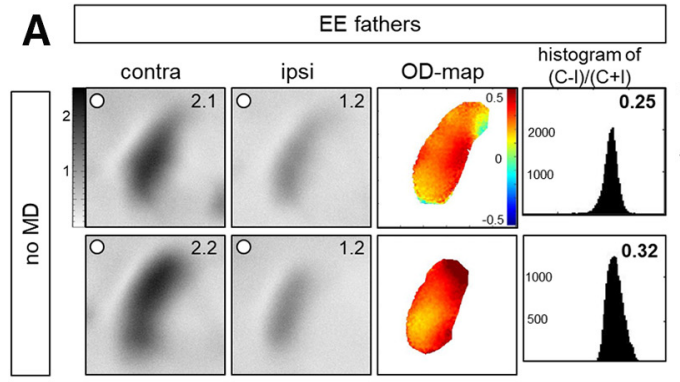

임

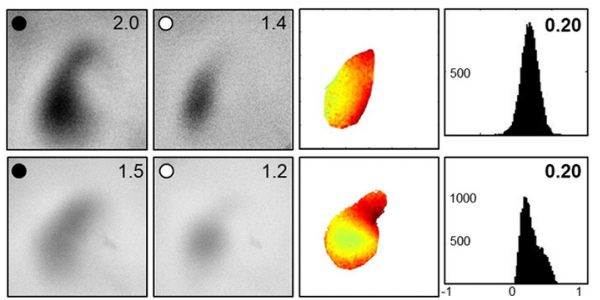

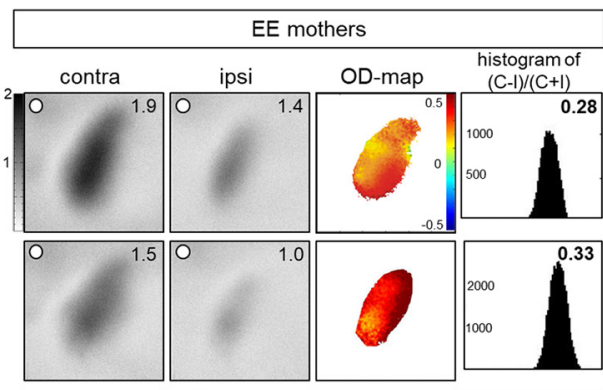

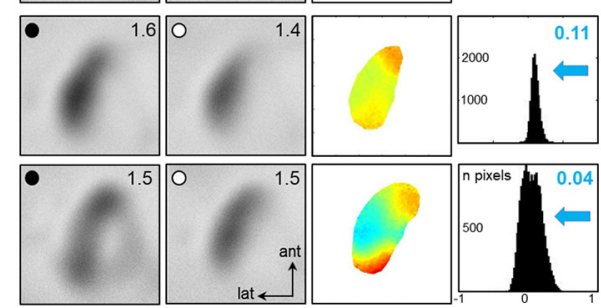

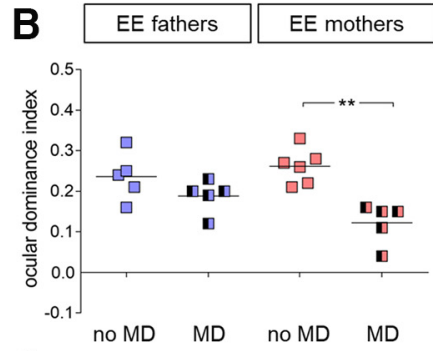

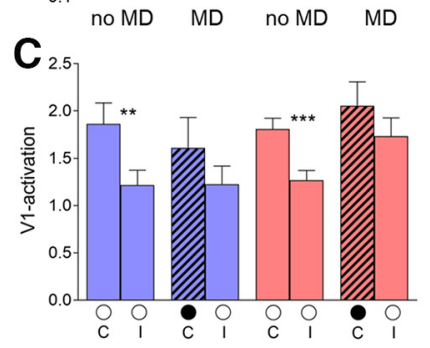

Figure 3 . Adult non-enriched offspring of EE-mothers display OD plasticity, but not non-enriched offspring of EE-fathers. $A$, Optically recorded activity maps elicited by visual stimulation of the contralateral (contra) or ipsilateral (ipsi) eye in the binocular part of V1 of SC-raised offspring of EE-fathers (left column) or EE-mothers (right column) without (no MD), and with $7 \mathrm{~d}$ of MD. Data display and quantification as in Figure 1. V1 activity maps from two individual animals of each condition (no MD/MD) are illustrated. In mice without MD, the activity patches evoked by visual stimulation of the contralateral eye are darker than those of the ipsilateral eye, warm colors (red) prevail in the two-dimensional OD maps and ODI values are positive. After MD in offspring of EE-fathers, V1 remained dominated by the deprived (contralateral) eye in, whereas offspring of EE-mothers displayed OD plasticity, i.e., both eyes activated V1 more equally strong and the ODI histogram shifted to the left (blue arrows). $\boldsymbol{B}, \boldsymbol{C}$, Quantification of visual cortical activation before and after MD: both ODI $(\boldsymbol{B})$ and V1 activation $(\boldsymbol{C})$ are illustrated. Statistical significance was calculated using ANOVA and $p$ values were corrected for multiple comparisons. $* * p<0.01$, $* * * p<0.001$.

increase of open eye responses in $\mathrm{V} 1$ was not significant $(p=0.052$, ANOVA), it is worth noting that the OD shift of the non-enriched mice born to EE-mother/SC-father pairs was clearly not mediated by a reduction of deprived eye responses in $\mathrm{V} 1$, as observed for the non-enriched pups of EE-parents (compare Figs. 2C, 3C, rightmost histogram) and for adult EE-mice (Fig. 1). Differences between no-MD mice of both groups were not significant $(p>0.05$, ANOVA).

Together, our data demonstrate that (1) in a primary sensory cortex, the plasticity promoting effect of EE can be transmitted to the next non-enriched generation, and (2) the life experiences of the mother have a greater impact on the continued V1 plasticity of the non-enriched SC offspring.

\section{Basic Visual abilities and enhanced optomotor reflex after md were similar in all experimental groups}

In addition to the assessment of OD plasticity, spatial vision of all experimental animals was measured daily using the virtual reality optomotor setup (Prusky et al., 2004). First, baseline spatial frequency and contrast sensitivity thresholds of the optomotor reflex were determined in all mice of the three major experimental groups (non-enriched SC-offspring of EE-parents or EE-father/ SC-mother or EE-mother/SC-father pairs).

Spatial frequency thresholds of adult non-EE mice born to EE-parents were $0.38 \pm 0.001 \mathrm{cyc} / \mathrm{deg}(n=11)$, values of mice born to EE-fathers were $0.38 \pm 0.001 \mathrm{cyc} / \mathrm{deg}(n$ $=11$ ), and the offspring of EE-mothers had a threshold of $0.39 \pm 0.001 \mathrm{cyc} / \mathrm{deg}(n=9)$; i.e., values did not differ significantly between the groups $(p=0.392$, ANOVA).
Baseline contrast sensitivity thresholds of the optomotor reflex were also determined for the three major mouse groups at six different spatial frequencies $(0.031,0.064$, $0.092,0.103,1.192$, and 0.272 cyc/deg): contrast sensitivity values were again similar for all the groups $(p>0.05$ for every spatial frequency and comparison, ANOVA).

Afterward, MD was performed in some of the animals of each experimental group and the spatial frequency and contrast sensitivity thresholds of the optomotor reflex were tested daily in the following $7 \mathrm{~d}$. As expected from previous research (Prusky et al., 2006), both spatial frequency and contrast thresholds of the open eye increased in all groups after MD. These data functionally confirmed that the MD eyes remained closed throughout the $7 \mathrm{~d}$ MD period, and served as an additional check for effective eye closure. In SC-mice born to EE-parents, the highest spatial frequency eliciting an optomotor response on Day 7 after MD was $0.45 \pm 0.003 \mathrm{cyc} / \mathrm{deg}(n=7)$, and thus significantly higher $(p=0.0011$, ANOVA) than the values before MD $(0.39 \pm 0.001 \mathrm{cyc} / \mathrm{deg}, n=5)$. In SC-mice born to EE-fathers, the spatial frequency threshold increased to $0.46 \pm 0.002 \mathrm{cyc} / \mathrm{deg}$ on Day 7 after MD $(n=5)$, significantly higher than the baseline value before MD (0.38 $\pm 0.001 \mathrm{cyc} / \mathrm{deg}, n=6 ; p=0.0007$, ANOVA). Likewise, in SC-mice born to EE-mothers, the threshold increased to $0.44 \pm 0.003 \mathrm{cyc} / \mathrm{deg}$ after MD $(n=5)$, whereas SC-offspring of EE-mothers without MD had a value of $0.39 \pm 0.002 \mathrm{cyc} / \mathrm{deg}$ on Day $7(n=5, p=$ 0.0043 , ANOVA; Fig. 4A). Spatial frequency thresholds of all MD-groups increased significantly from Day 0 to Day 7 $(p<0.001$ for EE-parents and EE-fathers, $p<0.01$ for EE-mothers, ANOVA). In more detail, thresholds in- 

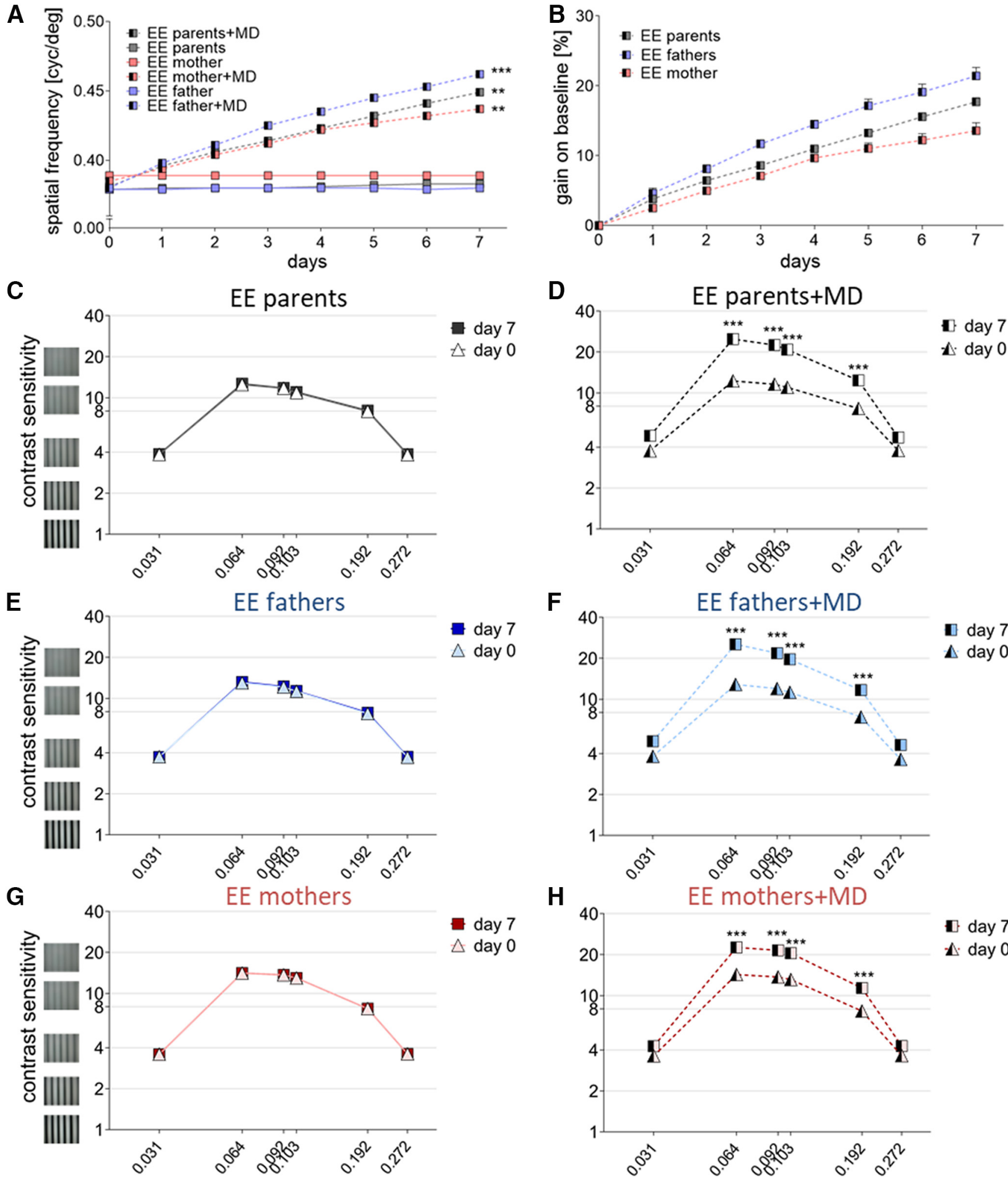

day 7

$\triangle$ day 0

spatial frequency [cyc/deg]

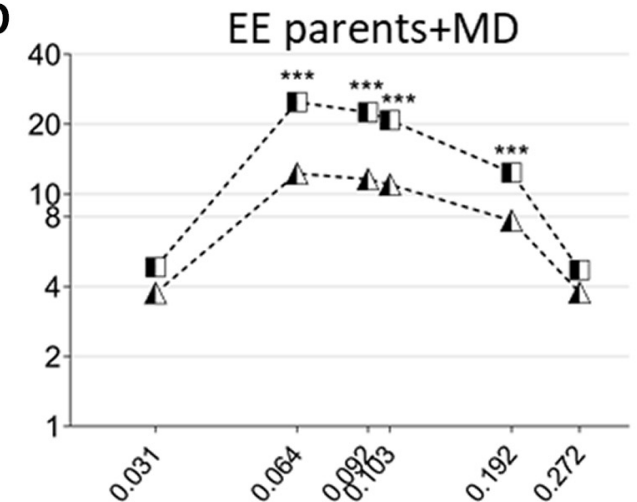

T. day 7

A. day 0

F

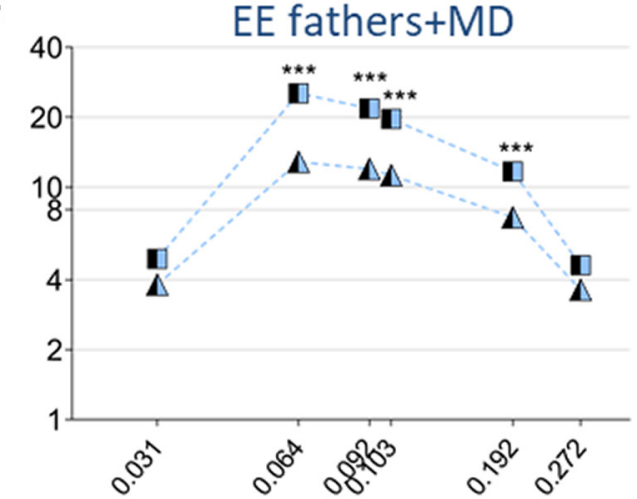

H

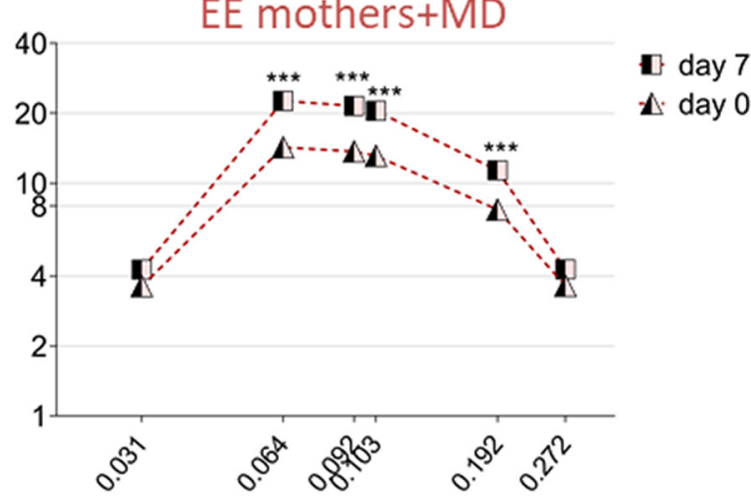

spatial frequency [cyc/deg]

Figure 4 . Experience-enabled improvements of the optomotor reflex where not different in non-enriched offspring of EE-parents, EE-fathers, and EE-mothers. $\boldsymbol{A}, \boldsymbol{B}$, The spatial frequency threshold in cyc/deg (A) and gain on baseline (in \%; $\boldsymbol{B}$ ) of the improvements of the optomotor reflex plotted as a function of days, over $7 \mathrm{~d}$ of MD or noMD. $\boldsymbol{C}-\boldsymbol{H}$, Contrast sensitivity thresholds of the optomotor reflex on days 0 and 7 after $\operatorname{MD}(\boldsymbol{D}, \boldsymbol{F}, \boldsymbol{H})$ or for the no MD-period $(\boldsymbol{C}, \boldsymbol{E}, \boldsymbol{G})$ at six different spatial frequencies. Note that both the spatial frequency and the contrast thresholds increased after MD in all three groups of non-enriched offspring. Statistical 
continued

significance was calculated using ANOVA and $p$ values were corrected for multiple comparisons. $* * p<0.01, * * * p<0.001$.

creased by $18 \%$, for SC-offspring of EE-parents, by $21 \%$ for SC-offspring of EE-fathers and by $14 \%$ for SCoffspring of EE-mothers (Fig. 4B). Without MD, spatial frequency thresholds remained constant over time $(p>$ 0.05, ANOVA).

Similarly, contrast sensitivity thresholds of all mice without MD remained constant over the $7 \mathrm{~d}$ of measurement ( $p>0.05$ for every frequency in each group, ANOVA; Fig. $4 C, E, G)$, whereas contrast sensitivity thresholds of the optomotor reflex of the open eye increased significantly after MD compared with Day 0 in all three groups of SC-offspring for 4 of 6 of the tested spatial frequencies. In detail, statistical analyses revealed the following $p$ values (ANOVA) comparing contrast thresholds on Day 0 before MD with Day 7 after MD for 0.031/0.064/0.092/0.103/ 0.192 and 0.272 cyc/deg): SC-offspring of EE-parents: $p$ $>0.05 / p<0.001 / p<0.001 / p<0.001 / p<0.01 / p>0.01$ (Fig. 4D), SC-offspring of EE-fathers $p>0.05 / p<0.001 / p$ $<0.001 / p<0.001 / p<0.01 / p>0.05$ (Fig. 4F), and SC-offspring of EE-mothers: $p>0.05 / p<0.001 / p<$ $0.001 / p<0.001 / p<0.01 / p>0.05$ (Fig. 4G). The values on Day 7 were not different between the three groups $(p>$ 0.05 for every frequency and group comparison, ANOVA; Table 3).

In summary, after MD, mice from all experimental groups showed similar increases both in spatial frequency and contrast sensitivity thresholds. Thus, in contrast to cortical OD plasticity, enhanced optomotor responses after MD do not seem to be modified by the rearing conditions of the animals' parents.

\section{Discussion}

There is growing evidence that the effects of an enriched environment can be long-lasting, and even include transmission of acquired features to the next generation (Arai and Feig, 2011). Surprisingly, we observed such a transgenerational transmission of increased neuronal plasticity in a primary sensory cortical area. After raising mice in EE cages, OD plasticity in V1 was not only preserved into old age, in contrast to SC-raised mice, but most interestingly also in their adult non-enriched offspring; i.e., in mice that never experienced enrichment directly. The OD plasticity visualized in V1 of adult nonenriched offspring of EE-parents was mechanistically similar to the OD plasticity of adult EE-mice. In both experimental groups, the OD shifts were primarily mediated by a reduction of V1 activation after visual stimulation of the (previously) deprived eye. Testing non-enriched SC-offspring of enriched mothers and non-enriched fathers versus enriched fathers and non-enriched mothers revealed that enriched mothers seem to have a greater contribution to the transgenerational effect. Whereas the adult offspring of enriched mothers and non-enriched fathers displayed OD plasticity after monocular deprivation, non-enriched offspring of enriched fathers and non-enriched mothers did not significantly change V1 activation despite the $7 \mathrm{~d}$ MD period. Notably, however, there is some mechanistic difference between the OD plasticity observed in EE-mice or non-enriched offspring of EE-parents and non-enriched offspring of EE-mothers/ nonEE-fathers. Although the OD shift of the former two experimental groups (EE-mice and SC-offspring of EEparents) is primarily mediated by a reduction of deprived eye responses in V1 after $7 \mathrm{~d}$ of MD, this was clearly not the case in the SC-offspring of EE-mothers: optical imaging rather revealed a small but insignificant $(p=0.052)$ increase in open eye V1 activation. Because a reduction of deprived eye responses in $\mathrm{V} 1$ after $7 \mathrm{~d}$ of $\mathrm{MD}$ is characteristic for OD plasticity in adult enriched mice (Greifzu et al., 2014), a similar kind of OD plasticity is transmitted to the next generation only if both parents are enriched. Thus, the life experiences of the father must also contribute to the offspring's cortical plasticity. Future in vivo spike recordings should reveal the mechanisms underlying these differences in OD plasticity, depending on whether only the mother or the father or both parents were raised in an enriched environment.

Together our data suggest that the supporting effect of EE on preserving OD plasticity into late adulthood can be passed on to the next generation, even if the offspring of EE-mice have never experienced EE-conditions on their own. Furthermore, the life experiences of the mother seem to have a greater impact on the continued OD plasticity of the offspring. We suggest that the increased OD plasticity observed in the non-enriched offspring of EE-parents may arise because of inherited epigenetic

Table 3 Contrast sensitivity values of mice born in SC from EE-parents, EE-father, or EE-mother on Day 7 of MD/no MD period

\begin{tabular}{lllllll}
\hline $\begin{array}{l}\text { Spatial frequency } \\
\text { (cyc/deg) }\end{array}$ & $\begin{array}{l}\text { EE-parents } \\
(\boldsymbol{n}=\mathbf{4})\end{array}$ & $\begin{array}{l}\text { EE-parents } \\
\mathbf{M D}\end{array}$ & $\begin{array}{l}\boldsymbol{( n = 7 )} \\
0.031\end{array}$ & $\begin{array}{l}\text { EE-fathers } \\
(\boldsymbol{n}=\mathbf{6})\end{array}$ & $\begin{array}{l}\text { EE-fathers } \\
\text { MD } \\
(\boldsymbol{n}=\mathbf{5})\end{array}$ & $\begin{array}{l}\text { EE-mothers } \\
\text { EE-mothers }\end{array}$ \\
0.064 & $3.8 \pm 0.35$ & $4.8 \pm 0.18$ & $3.7 \pm 0.08$ & $4.9 \pm 0.12$ & $3.6 \pm 0.04$ & $\begin{array}{l}\text { MD } \\
(\boldsymbol{n}=\mathbf{4})\end{array}$ \\
0.092 & $12.9 \pm 3.26$ & $24.9 \pm 1.12$ & $13.3 \pm 0.21$ & $25.9 \pm 0.51$ & $14.1 \pm 0.37$ & $22.6 \pm 0.27$ \\
0.103 & $11.9 \pm 2.64$ & $22.5 \pm 0.89$ & $12.3 \pm 0.20$ & $22.0 \pm 0.44$ & $13.7 \pm 0.38$ & $21.5 \pm 0.34$ \\
0.192 & $11.4 \pm 2.44$ & $20.8 \pm 0.69$ & $11.4 \pm 0.09$ & $20.2 \pm 0.31$ & $13.0 \pm 0.31$ & $20.5 \pm 0.38$ \\
0.272 & $8.2 \pm 1.31$ & $12.4 \pm 0.26$ & $7.7 \pm 0.12$ & $11.7 \pm 0.28$ & $7.7 \pm 0.15$ & $11.4 \pm 0.25$ \\
& $3.8 \pm 0.27$ & $4.7 \pm 0.11$ & $3.7 \pm 0.11$ & $4.7 \pm 0.09$ & $3.6 \pm 0.03$ & $4.3 \pm 0.03$
\end{tabular}

For the six different spatial frequencies tested the average contrast sensitivity for each group is listed as mean \pm SEM. 
changes and modified behavior, e.g., mother-pup interactions.

Epigenetic changes have been shown to affect the neural development of the offspring in previous studies. It was reported that pregnant rats exposed to enriched environment showed enhanced ability to find their way in a maze as well as the ability of their offspring to do the same (Kiyono et al., 1985). In another study, female mice exposed to EE showed enhanced learning ability that was transmitted to their offspring even if the exposure to $\mathrm{EE}$ was before pregnancy (Dell and Rose, 1987). Their observations were similar when non-EE foster mothers raised the offspring of EE-mothers, suggesting that the effect of EE was transmitted to the offspring before birth, presumably in utero. In support of these data, Arai et al. (2009) showed that 4-week-old offspring of EE-mice displayed enhanced synaptic plasticity in the hippocampus, as their EE-raised parents. Interestingly, only the mother's contribution to the transgenerational transmission of the EE-effect was significant. The enhanced hippocampal plasticity was also observed when the offspring of EEmothers were raised from birth by a non-EE foster mother, suggesting that the effect of $\mathrm{EE}$ was transmitted to the offspring before birth (Arai et al., 2009). It is possible that in utero exposure to EE had a plasticity promoting effect also in our study; however, because the OD shifts in offspring of EE-parents and EE-mothers were mechanistically different, in utero exposure is likely only a contributing factor and cannot be accounted for all the observed effects. Future experiments with non-EE foster mothers should clarify whether the prolonged OD plasticity is because of specific behavior of an enriched mother or due to in utero epigenetic modifications. In this context, it would also be interesting to investigate the expression profile and the chromatin remodeling of plasticity-related genes after exposure to EE and to test whether any modifications can be transmitted to the next generation.

It is well known that rodent mothers play a big role for both prenatal and postnatal development of their offspring (Liu et al., 2000). Mothers provide nutritional and behavioral support by, e.g., licking and grooming, which may have long lasting beneficial effects for brain plasticity (Champagne et al., 2003). Furthermore, mothers raised in EE provide their pups with higher levels of maternal care compare to SC-raised mothers, which may also explain the long-lasting plasticity observed in their offspring (Baroncelli et al., 2010). During the first 2 weeks of life, rodents stay in the nest, totally depending on the mother, which is the most important source of sensory experience (Ronca et al., 1993). EE-pups receive higher levels of maternal care, continuous physical contact, and higher levels of licking compared with SC-reared pups (Sale et al., 2004). It has been proposed that higher maternal care in EEpups affects their visual system development (Cancedda et al., 2004), possibly because of increased BDNF levels in the offspring caused by variations in maternal care (Liu et al., 2000). Mimicking part of maternal behavior during the first $10 \mathrm{~d}$ of a rat pup's life by using tactile stimulation (massage), a procedure previously shown to compensate for the negative effects of maternal deprivation (Schan- berg and Field, 1987), was able to reproduce the EEdependent acceleration of visual development in rat pups born in SCs (Guzzetta et al., 2009). The effect of tactile stimulation was attributed to increased IGF-I levels in P18 pups, as blocking IGF-I abolished the EE-induced acceleration of visual system development (Guzzetta et al., 2009). IGF-I signaling could therefore be a contributing factor to the continued OD plasticity of EE-mice and their non-enriched offspring observed in the present study.

Furthermore, the level of maternal care can also influence the response to stress in adulthood (Meaney, 2001). High levels of maternal care results in elevated levels of hippocampal serotonin in the pups, leading to increased expression of the transcription factor NGFI-A binding to the glucocorticoid (GR) promoter. Higher levels of NGFI-A result in increased expression of the GR-receptor through posttranslational modifications like DNA hypomethylation and histone acetylation which is correlated with reduced stress levels. These epigenetic modifications are maintained into adulthood and are heritable, marking the level of maternal care on gene expression patterns across generations (Weaver et al., 2004).

Although the mother's role in the fitness of offspring has been long recognized, there is also increasing literature on epigenetic transmission of neurobehavioral phenotypes through the paternal line (for review, see Yuan et al., 2016). The fact that the OD shift in the offspring of EEmothers and SC-fathers was not the same as in the offspring of EE-parents (or in adult EE-mice) already indicates that the transgenerational transmission of OD plasticity cannot solely be explained by any epigenetic/ behavioral changes of mothers, but that epigenetic transmission through the paternal and/or maternal line must also be involved. Therefore, epigenetic influences and modified maternal care likely work in concert to support the transgenerational transmission of the increased OD plasticity.

In addition to imaging cortical OD plasticity, we assessed the basic visual capabilities of mice by measuring the spatial frequency and contrast thresholds in the optomotor setup before and during monocular deprivation. Neither of the measured parameters differed between the three major experimental groups (EE-parents, EEmothers/SC-fathers, EE-fathers/SC-mothers) before or during the MD, and values were also similar to previously published data from similarly aged SC- and EE-mice (Prusky et al., 2006; Greifzu et al., 2014). Although it was shown that EE has a positive effect on early visual system development (Cancedda et al., 2004), we did not observe any enhancement in the optokinetic reflexes in any of the examined groups.

The observation that MD-induced optomotor enhancements do not depend on the raising conditions of the mice or their parents suggests that life experiences affect the underlying neuronal circuits differently compared with $\mathrm{OD}$ plasticity. It is known that the optomotor response is mediated by the brainstem accessory optic system, whereas cortical circuits are necessary for the MDinduced increases in optomotor thresholds (Prusky et al., 2006). Specifically, it was recently shown that cortico- 
fugal projections from V1 to the accessory optic system, in particular to the optic tract and dorsal-terminal nuclei (NOT-DTN), underlie learning-induced optokinetic reflex potentiation (Liu et al., 2016). Thus, experience-induced optomotor enhancements and OD plasticity in V1 depend on different nerve cell circuits. The results of these two "plasticity" paradigms can therefore be independent of each other and must not follow similar rules. In fact, it was shown previously that results can dissociate: whereas ibuprofen treatment could rescue the deprivation-induced enhancement of optomotor thresholds in mice with a cortical stroke in the primary somatosensory cortex, it did not rescue OD plasticity in the same animals (Greifzu et al., 2011).

Genetic programs, epigenetic information, and experience work in concert to optimize the development and behavior of an organism for survival in a particular environment. There is now ample evidence that, in addition to the genes transferred to the next generations by germ lines, experiences of the parents can markedly influence both structure and function of the nervous system and behavior of subsequent generations through inherited epigenetic modifications (Arai and Feig, 2011). Our work provides additional support to this idea by showing that even in a primary sensory cortical area, a plasticity-promoting effect of an enriched environment, or less-deprived rearing, can be transferred to the next generation. Most likely based on the important role of the mothers in prenatal and early postnatal development of the offspring, mothers appear to have a slightly more important role than fathers in this transgenerational transmission. Importantly, our data further indicate that the outcome of any brain plasticity experiment might not only depend on the life experiences of the particular experimental animal but also can be influenced by the life experiences of the parents of this experimental animal, which therefore should be carefully documented. Further investigations are necessary to elucidate the exact mechanisms underlying this transgenerational rescue of brain plasticity and the differences in the magnitude and mechanisms of the experience-dependent V1 activation changes.

\section{References}

Arai JA, Feig LA (2011) Long-lasting and transgenerational effects of an environmental enrichment on memory formation. Brain Res Bull 85:30-35. CrossRef Medline

Arai JA, Li S, Hartley DM, Feig LA (2009) Transgenerational rescue of a genetic defect in long-term potentiation and memory formation by juvenile enrichment. J Neurosci 29:1496-1502. CrossRef Medline

Baroncelli L, Sale A, Viegi A, Maya-Vetencourt JF, De Pasquale R, Baldini S, Maffei L (2010) Experience-dependent reactivation of ocular dominance plasticity in the adult visual cortex. Exp Neurol 226:100-109. CrossRef Medline

Cancedda L, Putignano E, Sale A, Viegi A, Berardi N, Maffei L (2004) Acceleration of visual system development by environmental enrichment. J Neurosci 24:4840-4848. CrossRef Medline

Cang JH, Kalatsky VA, Löwel S, Stryker MP (2005) Optical imaging of the intrinsic signal as a measure of cortical plasticity in the mouse. Vis Neurosci 22:685-691. CrossRef Medline

Champagne FA, Francis DD, Mar A, Meaney MJ (2003) Variations in maternal care in the rat as a mediating influence for the effects of environment on development. Physiol Behav 79:359-371. CrossRef Medline

Dell PA, Rose FD (1987) Transfer of effects from environmentally enriched and impoverished female rats to future offspring. Physiol Behav 39:187-190. CrossRef Medline

Diamond MC, Krech D, Rosenzweig MR (1964) The effects of an enriched environment on the histology of the rat cerebral cortex. $J$ Comp Neurol 123:111-120. CrossRef Medline

Dräger UC (1978) Observations on monocular deprivation in mice. J Neurophysiol 41:28-42. CrossRef Medline

Espinosa JS, Stryker MP (2012) Development and plasticity of the primary visual cortex. Neuron 75:230-249. CrossRef Medline

Gordon JA, Stryker MP (1996) Experience-dependent plasticity of binocular responses in the primary visual cortex of the mouse. $J$ Neurosci 16:3274-3286. CrossRef Medline

Greifzu F, Kalogeraki E, Löwel S (2016) Environmental enrichment preserved lifelong ocular dominance plasticity, but did not improve visual abilities. Neurobiol Aging 41:130-137. CrossRef Medline

Greifzu F, Pielecka-Fortuna J, Kalogeraki E, Krempler K, Favaro PD, Schlüter OM, Löwel S (2014) Environmental enrichment extends ocular dominance plasticity into adulthood and protects from stroke-induced impairments of plasticity. Proc Natl Acad Sci U S A 111:1150-1155. CrossRef Medline

Greifzu F, Schmidt S, Schmidt KF, Kreikemeier K, Witte OW, Löwel $S$ (2011) Global impairment and therapeutic restoration of visual plasticity mechanisms after a localized cortical stroke. Proc Natl Acad Sci USA 108:15450-15455. CrossRef Medline

Guzzetta A, Baldini S, Bancale A, Baroncelli L, Ciucci F, Ghirri P, Putignano E, Sale A, Viegi A, Berardi N, Boldrini A, Cioni G, Maffei $L$ (2009) Massage accelerates brain development and the maturation of visual function. J Neurosci 29:6042-6051. CrossRef Medline

Kalatsky VA, Stryker MP (2003) New paradigm for optical imaging: temporally encoded maps of intrinsic signal. Neuron 38:529-545. Medline

Kiyono S, Seo ML, Shibagaki M, Inouye M (1985) Facilitative effects of maternal environmental enrichment on maze learning in rat offspring. Physiol Behav 34:431-435. CrossRef Medline

Lehmann K, Löwel S (2008) Age-dependent ocular dominance plasticity in adult mice. Plos One 3:e3120. CrossRef Medline

Liu D, Diorio J, Day JC, Francis DD, Meaney MJ (2000) Maternal care, hippocampal synaptogenesis and cognitive development in rats. Nat Neurosci 3:799-806. CrossRef Medline

Liu B, Huberman AD, Scanziani M (2016) Cortico-fugal output from visual cortex promotes plasticity of innate motor behaviour. Nature 538:383-387. CrossRef Medline

Löwel S, Dehmel S, Makowiecki K, Kalogeraki E (2017) Environmental conditions strongly affect brain plasticity. eNeuroforum 24: A19-A29. CrossRef

Meaney MJ (2001) Maternal care, gene expression, and the transmission of individual differences in stress reactivity across generations. Annu Rev Neurosci 24:1161-1192. CrossRef Medline

Prusky GT, Alam NM, Douglas RM (2006) Enhancement of vision by monocular deprivation in adult mice. J Neurosci 26:11554-11561. CrossRef Medline

Prusky GT, Alam NM, Beekman S, Douglas RM (2004) Rapid quantification of adult and developing mouse spatial vision using a virtual optomotor system. Invest Ophthalmol Vis Sci 45:46114616. CrossRef Medline

Ronca AE, Lamkin CA, Alberts JR (1993) Maternal contributions to sensory experience in the fetal and newborn rat (Rattus norvegicus). J Compar Psychol 107:61-74. CrossRef Medline

Rosenzweig MR, Krech D, Bennett EL, Diamond MC (1962) Effects of environmental complexity and training on brain chemistry and anatomy: a replication and extension. J Comp Physiol Psychol 55:429-437. CrossRef Medline 
Sale A, Berardi N, Maffei L (2014) Environment and brain plasticity: towards an endogenous pharmacotherapy. Physiol Rev 94:189234. CrossRef Medline

Sale A, Putignano E, Cancedda L, Landi S, Cirulli F, Berardi N, Maffei $L$ (2004) Enriched environment and acceleration of visual system development. Neuropharmacology 47:649-660. CrossRef Medline

Sale A, Maya-Vetencourt JF, Medini P, Cenni MC, Baroncelli L, De Pasquale R, Maffei L (2007) Environmental enrichment in adulthood promotes amblyopia recovery through a reduction of intracortical inhibition. Nat Neurosci 10:679-681. CrossRef Medline

Schanberg SM, Field TM (1987) Sensory deprivation stress and supplemental stimulation in the rat pup and preterm human neonate. Child Dev 58:1431-1447. CrossRef Medline van Praag H, Kempermann G, Gage FH (2000) Neural consequences of environmental enrichment. Nat Rev Neurosci 1:191-198. CrossRef Medline

Weaver ICG, Cervoni N, Champagne FA, D'Alessio AC, Sharma S, Seckl JR, Dymov S, Szyf M, Meaney MJ (2004) Epigenetic programming by maternal behavior. Nat Neurosci 7:847-854. CrossRef Medline

Wiesel TN, Hubel DH (1963) Effects of visual deprivation on morphology and physiology of cells in the cat's lateral geniculate body. J Neurophysiol 26:978-993. CrossRef Medline

Yuan TF, Li A, Sun X, Ouyang H, Campos C, Rocha NBF, AriasCarrión O, Machado S, Hou G, So KF (2016) Transgenerational inheritance of paternal neurobehavioral phenotypes: stress, addiction, ageing and metabolism. Mol Neurobiol 53:6367-6376. CrossRef Medline 